\title{
Edouard Pichon et les patois
}

\author{
Gabriel Bergounioux ${ }^{1 *}$ \\ ${ }^{1}$ Université d'Orléans (LLL - UMR 7270)
}

\begin{abstract}
Résumé. Mobilisé comme médecin pendant la Première guerre mondiale, Edouard Pichon est resté en relation épistolaire avec son oncle et collaborateur pour l'Essai de grammaire de la langue française, Jacques Damourette. Dans une série de missives de l'année 1915, il lui fait part des études qu'il mène sur les dialectes et les langues régionales. A partir de listes de mots et de paradigmes de conjugaison, il cherche à retrouver dans les formes du picard, du franco-provençal, du catalan et du languedocien la preuve d'une permanence de la mentalité française à travers ses différents parlers. Mettant à contribution ce qu'il entend chez les habitants du Santerrois et sollicitant comme informateurs les militaires des unités dans lesquelles il est affecté, il revient à une approche philologique en étudiant le provençal dans Mirèio de Mistral. Utilisant une notation phonétique idiosyncrasique, Pichon a une approche différentialiste. Il conçoit les dialectes comme l'interprétation prosodique, "affective", d'une même langue, le français, dont la fonction «représentative » resterait constante par l'effet d'un atavisme racial. Ainsi la grammaire devient l'illustration d'une théorie qui fait des langues, et des rapprochements entre elles, la preuve d'une transcendance des propriétés de race sur les formations sociales. S'il y a des affinités entre le français, l'occitan, le breton et l'alsacien, ce n'est pas un effet de contact mais la rémanence d'un substrat biologique commun. A ce titre, Pichon s'oppose à la vision historiciste de Gaston Paris en romanistique comme aux principes sociologiques d'Antoine Meillet.
\end{abstract}

Abstract. Edouard Pichon and the "patois". Mobilized as a doctor during the First World War, Edouard Pichon kept in touch by mail with his uncle Jacques Damourette, the co-author for their Essai de grammaire de la langue française. In his letters of the year 1915, he tells him about his studies of dialects and regional languages. Using lists of words and conjugation paradigms, he seeks to find in the forms of Picard, FrancoProvençal, Catalan and Languedocian the proof of a permanence of the French mentality through these different speeches. Drawing on what he hears from the inhabitants of the Santerrois and soliciting various soldiers as informants, he preferred a philological method to study Provençal in Mistral's Mirèio. Using an idiosyncratic notation of sounds, Pichon has a differentialistic approach. He sees dialects as the prosodic interpretation of the same language, French, as an "affective" melody whose "representative" function would remain constant by the effect of racial atavism. Thus, grammar becomes an illustration of a theory that makes languages, and links between them, the proof of a transcendence of the race on social formations.

* Corresponding author : gabriel.bergounioux@univ-orleans.fr 
If there are affinities between French, Occitan, Breton and Alsatian, it is not the result of contact but the persistence of a common biological substrate. In this respect, Pichon opposes Gaston Paris's historicalist vision of Romance languages and Antoine Meillet's sociological principles.

L'œuvre linguistique d'E. Pichon (1890-1940) tranche singulièrement parmi les ouvrages de son temps [Portine 1996; Muni Toke 2013]. D'abord, Les attendus politiques et psychanalytiques de l'auteur, si paradoxaux, n'étaient guère répandus dans le champ de la linguistique. Ensuite, au sein d'une discipline qui s'est professionnalisée dans l'enseignement supérieur depuis les années 1860, Pichon et son oncle et collaborateur, Jacques Damourette, restent des horsains, des amateurs. Leur Essai de grammaire de la langue française, ou plutôt Des mots à la pensée puisque tel est son premier titre, n'a pas de véritable équivalent par ses dimensions: les seize volumes de l'Histoire de la langue française de F. Brunot [1905-1937] ou les six tomes de la Grammaire Historique de la langue française de K. Nyrop [1899-1930] ne justifient leur pagination qu'à s'inscrire dans une perspective diachronique. Enfin, la terminologie est réputée pour son ésotérisme sans concession: environ trois cent cinquante termes figurent dans le glossaire, sans ménagement pour la nomenclature traditionnelle.

Au nombre des mérites reconnus à Des mots à la pensée, il y a l'intérêt déclaré pour les formes de l'oral cueillies au vol et données en exemples comme autant d'attestations admissibles de l'usage, avec référence anonymisée des témoins. Cette prise en compte de la variation diastratique (ou diaphasique) s'est étendue, ce qu'on sait moins, à la variété diatopique, aux «patois » dans la conception qui prédominait à l'époque. Cette contribution présente une correspondance dans laquelle E. Pichon, écrivant à J. Damourette, lui fait part de ses recherches et de ses avis en matière de dialectologie.

\section{Lettres de Pichon sur les patois}

\subsection{Le statut des patois}

Pichon est à sa façon une incarnation de l'amour de la langue [Milner 1978]. La suprématie affirmée du français justifiait le programme de francisation scolaire qui éliminait les langues régionales qui représentaient pourtant une forme d'expression du génie populaire :

Ceci nous amène à distinguer deux ordres de différence entre les parlers dérivés d'un même idiome. Appelons cet idiome l'idiome originel. Aux parlers qui en sont dérivés, nous donnons le nom de dialectes. Parmi ces dialectes, il en est qui ne présentent entre eux que des différences peu profondes qui naissent dans un ensemble humain de race homogène usant du même parler. (...) Les différences de ce premier ordre ne restent jamais que fort petites (...). Un tel groupe de dialectes s'appelle une lingualité et les différences de la même lingualité s'appellent couramment différences dialectales. L'unité de la lingualité résulte en somme de l'identité de réaction du peuple à la langue héritée ou acceptée par lui. Entre deux dialectes pris dans deux lingualités différentes, se voient les différences du second ordre, celles qui procèdent de l'origine ethnique différente des peuples ayant parlé l'idiome originel (...). Nous leur donnons le nom de divergences idiomatiques. [Damourette \& Pichon $1930: 19]$

Ainsi, pour une langue donnée, le «dialecte» correspondrait à la transformation diachronique d'une langue par la race qui la parle depuis l'origine alors que l' « idiome » résulterait des changements qu'introduit une race allophone dans la langue qu'elle emprunte. Paradoxalement, l'affirmation du primat des races sur l'usage des langues constituait une critique du dogme comparatiste qui, se fondant sur une hypothèse migratoire, superposait à l'extension linguistique l'expansion ethnique. 
Et, plus loin :

Les patois ne vivent donc auprès des langues que dans un véritable état de servitude, et le terme naturel de cette évolution, c'est leur mort. En effet, outre l'influence qu'elle exerce sur les patois, outre l'emprise absolue qu'elle prend sur la pensée des élites dans les pays à patois, la langue arrive à étendre de plus en plus le domaine dans lequel elle est le parler unique (...). [Ibid. : 24]

Les patois constitueraient un moment dans l'histoire d'une langue, une période de morcellement et de stagnation intellectuelle qui trouve sa conclusion au moment où Pichon consigne ses commentaires.

\subsection{Présentation des lettres}

Voici la description, dans le catalogue du libraire Julien Mannoni, des documents traitant de ce sujet :

B) 13 longues lettres autographes signées (2 incomplètes) à son oncle Jacques Damourette, datées du 17 juillet au 16 novembre 1915 (46 pp. in-12 et in-16) : études des patois (Pichon est alors mobilisé et donc confronté à toutes sortes de « parlures ») et de lexicographie ; C) manuscrits divers, certains signés et datés : " exposé de la conjugaison provençale mistralienne considérée en soi » (4 pp. in-16), « notes sur le patois haut-alsacien » $(2 \mathrm{pp}$. in-12) [...]), " patois de Nauroy et de Beauvais, canton du Catelet » (3 pp. in-8 dans un cahier), " provençal » (8 pp. in- 8 dans un cahier) [...]. E) Lettre tapuscrite signée de Paul Lebel à Hélène Pichon à propos de l'article : il a parlé du travail de Fernand Pichon à M. Dauzat et celui-ci lui a conseillé de s'adresser à Charles Bruneau, qui pourrait le faire passer dans la «Revue de linguistique romane » [...]. Dans son avertissement, Edouard Pichon explique que Fernand Pichon vécut à Gamay (où son père avait des vignes) de 1860 à 1886 et qu'il rédigea ses notes «vers 1903-1905, époque à laquelle feu Jean Mongin (...) venait me donner le goût de la linguistique et de la dialectologie, et où mon père voulut me faire plaisir en m'apprenant son patois. »

Ensemble disparate, réuni par le hasard des legs et des ventes, concentré sur une douzaine d'années (de 1903 à 1915), ces essais précèdent d'une quinzaine d'années la parution de la grammaire et la notoriété psychiatrique de Pichon.

Les quatorze lettres (et non treize comme annoncées) ont été écrites de juillet à novembre 1915. E. Pichon, qui avait fait ses classes peu avant la guerre à Hirson, a déféré à l'ordre de mobilisation et rejoint son unité sur le front, affecté dans les hôpitaux militaires, en retrait de la ligne de combat. La correspondance, censure oblige, ne porte pas d'indication de lieu en en-tête. On lit dans la missive datée du 16 août : «(...) Avant de quitter Rosières », une commune de la Somme où Pichon était caserné en juillet. L'affectation auprès du $96^{\mathrm{e}}$ régiment d'infanterie, mentionnée dans des correspondances du 30 septembre et du 25 octobre, permettrait de le situer ensuite quelque part en Champagne, au moment des deux grandes offensives tentées par Joffre à cette date. Malgré la violence de l'affrontement, Pichon semble disposer d'un loisir suffisant pour lire, essayer d'apprendre le provençal en lisant Mirèio et poursuivre une relation épistolaire suivie avec son oncle.

\subsubsection{Inventaire}

Sauf mention contraire, les lettres ont un format de $11 \times 17 \mathrm{~cm}$ environ. Après la date, Pichon a souvent précisé à son oncle les courriers auxquels il répondait. Les deux lettres sans date, parce qu'il manque le premier feuillet, ont été intercalées à l'endroit le plus probable.

- Lettre $1 \mathrm{du} 17$ juillet (réponse à une lettre du 12 juillet) 4 pages 
- Lettre 2 du 25 juillet (réponse à une lettre du 21 juillet)

8 pages + un plan

carte dessinée de l'Europe centrale avec légende au verso

- Lettre 3 du 29 juillet (réponse à une lettre du 26 juillet)

- Lettre 4 du 31 juillet (réponse à une lettre du 28 juillet)

- Lettre 5 (incomplète, non datée)

- Lettre 6 (incomplète, non datée) format $14 \times 18 \mathrm{~cm}$

- Lettre 7 du 16 août (réponse à une lettre du 9 août)

- Lettre 8 du 30 septembre (réponse aux lettres du 22 et 24 septembre) carte de correspondance militaire

- Lettre 9 du 10 octobre (réponse à une lettre du 4 octobre)

- Lettre $10 \mathrm{du} 16$ octobre

2 pages

4 pages

2 pages

4 pages

2 pages

1 page

4 pages

2 pages

1 page

format $11 \times 14 \mathrm{~cm}$, carte de correspondance militaire

- Lettre 12 du 4 novembre (réponse aux lettres du 29 et 31 octobre)

- Lettre 13 du 11 novembre (réponse aux lettres du 5 et du 7 novembre)

- Lettre 14 du 16 novembre (réponse à une lettre du 12 novembre) format $11 \times 13,5 \mathrm{~cm}$

4 pages

3 pages

6 pages

Toutes les lettres sont manuscrites, d'une écriture serrée et facilement lisible. Les références seront faites de façon conventionnelle en indiquant « $\mathrm{L} 1$ » pour la première lettre, « L2 » pour la deuxième, etc.

\subsubsection{Contenu de la correspondance}

Le plus remarquable dans ces échanges est la quasi-absence de notations concernant la vie quotidienne. Pichon ne fait jamais allusion à ses conditions de travail et les soldats autour de lui n'apparaissent qu'à titre d'informateurs pour ses enquêtes linguistiques. Si l'on a conservé pour cette étude les éléments d'ordre philologique, voici quels sont les autres sujets abordés en recensant, précédés du signe + , les paragraphes consacrés au français :

L1 (4409 signes)

- réponse à une proposition de J. Damourette d'adopter une datation gauloise (542 signes) ;

- souhait d'une inclusion des fortifications de la rive droite du Rhin au moment de l'annexion de la rive gauche du Rhin à la fin de la guerre (942 signes) ;

+ sur le comparatif en français (371 signes).

L2 (13137 signes)

- dissertation sur l'Europe centrale et la place des différents peuples slaves dans le découpage à venir des frontières, du nord des Balkans à la Baltique (9829 signes + carte).

L3 (2598 signes)

+ sur le subjonctif et sur les degrés de comparaison (803 signes).

L4 (5760 signes)

- nomination de Jean Weber au Journal (1426 signes);

+ sur la prononciation des lettres quiescentes (1424 signes).

L5 (1727 signes) - incomplète

L6 (4340 signes) - incomplète

- récit de rêve (615 signes) ;

- rappel des revendications françaises à formuler au moment de la signature de la paix avec l'Allemagne (375 signes).

L7 (2574 signes)

- congratulations de Pichon à Damourette qui a contracté un engagement bénévole dans un hôpital parisien (341 signes). 


\section{L8 (1818 signes)}

+ sur la classe strumentale et la locution « histoire de » (936 signes).

L9 (7941 signes)

- réflexions sur les frontières de la future Serbie (1704 signes).

L10 (2432 signes)

- commentaires accompagnant l'envoi d'une pièce jointe (qui ne figure pas dans le lot)

concernant la « race française » (742 signes) ;

- activités hospitalières de Damourette à «l'hôpital 106 » rue Armand-Moisant et de

Pichon au front (499 signes) ;

- demande d'information sur la démission de Delcassé (245 signes) ;

- demande d'envoi d'une carte de la région de Verdun - Vouziers (134 signes).

L11 (2486 signes)

- sur la prononciation de « Marinllard» (299 signes).

L12 (5460 signes)

- anthropologie des races (4021 signes);

- commentaire d'un article publié par J. Weber dans Le Journal (600 signes).

L13 (4720 signes)

- anthropologie raciale et craniologie du Proche Orient (1291 signes) ;

+ étymologie de rafistoler (325 signes).

L14 (9187 signes)

+ considérations sur l'usage de à, dans et lors (2236 signes).

Ont été conservées, dans le calcul global des signes, les formules initiales et finales, la date etc. La multiplication des tirets, des points etc., les mots rayés et le décompte des espaces font que les chiffres avancés doivent être retenus comme des ordres de grandeur. Sur un peu moins de 70000 signes au total (68589 exactement), 25000 environ ne concernent pas directement des questions linguistiques. Le partage entre ce qui est d'ordre linguistique et ce qui ne l'est pas reste discutable puisque, dans la perspective adoptée par Pichon, les considérations de race et de langue sont liées quoique non confondues. Au total, les éléments concernant les patois représentent la moitié de cette correspondance.

\section{Quand Pichon parle des patois}

En première approche, on peut distinguer trois thèmes de réflexion qui coïncident très largement avec la succession des affectations :

- la description des patois d'oïl septentrionaux (à Rosières),

- une mention des dialectes franco-provençaux,

- la découverte de l'occitan (provençal) et du catalan (en Champagne).

\subsection{Parlers du domaine picard}

Les relevés s'étendent du 17 juillet au 16 août 2015. L'essentiel résulte d'informations fournies par l'abbé Dourlen et d'observations sur les habitants de Rosières, en particulier « le père » et « la mère Doublet » chez qui le jeune médecin est logé.

\subsubsection{Les désignations.}

L1 (2312 signes)

« picard », « santerrois », « dialectes parlers d'oui ».

L2 (2474 signes)

« santerrois », « forme picarde », « franco-picard ».

L3 (1080 signes)

« santerrois », rosiérois », « analogie picarde », [« bourguignon »].

L4 (2576 signes) 
« picard », « patois de l'Arrière-Picardie » = « Arrouaise ».

\section{L5 (1554 signes)}

« picards ».

L6 (1761 signes)

«Valenciennes ».

\section{L7 (1564 signes)}

« rosiérois ».

A côté de désignations géographiques où «picard» domine, on relève l'évitement du terme « dialectes », rayé et remplacé par «parlers » avec cette remarque en incise :

Je sais maintenant pourquoi mon hôtesse et son mari parlent si purement le picard et si peu le français (que d'ailleurs ils comprennent parfaitement: cf. les nègres qui parlent leur dialecte natal quoique comprenant les dialectes environnants) : c'est qu'ils ne savent pas lire. (L1)

L'usage de Pichon oscille entre les considérations des dialectologues et la pratique philologique d'analyse de l'ancien français avec un découpage de la langue d'oïl en cinq ensembles (ou plus) - dont le bourguignon - afin de localiser les manuscrits. Ceux-là se conformaient aux propositions des directeurs de Romania (fondée en 1872), Paul Meyer et Gaston Paris, qui insistaient sur l'atomisation des parlers pour argumenter l'existence d'un continuum gallo-roman [Paris 1888]. A côté du «picard» sont spécifiées des aires d'extension plus restreinte (Santerre, Arrouaise), jusqu'à l'échelle de la commune (Valenciennes, Rosières-en-Santerre).

On relève que, se référant à des «parlers d'oui », Pichon entérine la séparation oc/oïl en quoi il se démarque de Romania. Quand il qualifie une forme de «compromis francopicard » (L2), on ne sait s'il désigne par là un phénomène de calque ou une forme de transition.

\subsubsection{Les études}

\section{Lexique}

Indépendamment de toute considération phonétique ou grammaticale, il y a un relevé au vol des correspondants « santerrois » des mots français chez, le soir, le fils, petit (L1), tendre, de nuit, ouvrable, blanc d'œuf, crible, radis, manquer (L2), peu (L3), le long de, chaud, coulisse, pleurer, couteau, coupe plus les noms de nombre (L4) et penser (L5). Sont jointes de rares remarques étymologiques à propos d'une préposition (mon de < «maison de »= chez, L1) ou un nom de lieu (Caours < Casa Ursi = maison de l'ours, L4) et quelques locutions :

Pour dire qu'un homme a sept frères et sœurs, l'on dit ici qu'il est « né à huit », et cette expression elliptique, comparable à « nous deux Jacques », me plaît beaucoup par sa brièveté, sa concision. (L1)

Dans L6, Pichon transmet à son oncle une liste de trente-deux mots qui témoignent d'un premier souci d'inventaire thématique : onze noms d'animaux domestiques, douze noms de fruits et légumes...

\section{Phonétique}

A l'exception d'une observation sur le traitement des palatales :

$(\ldots)$ en ce «tch» si fréquent dans certains parlers de bien des pays de France. On dit bien « un quevau » kvo, mais, en dit alors qu'à Valenciennes on un dit un khẽ, « quien »; ici, c'est « un tchein », tchẽ, et le derrière s'appelle « che tchiul », cýtchu. (L6)

toutes les remarques portent sur la réalisation des voyelles rapportées à leur correspondant en français.

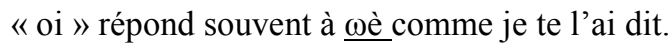




\begin{tabular}{|c|c|}
\hline $\begin{array}{c}\text { Ex. : foire } \\
\text { moison } \\
\text { voirre }\end{array}$ & 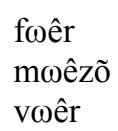 \\
\hline
\end{tabular}

Mais dans l'imparfait, oi répond à $\omega \underline{e}$, et même, dans la bouche de certaines gens dont mon hôtesse la mère Doublet, cet e est si fermé qu'on entend presque $\omega i$, ce qui fait enfin comprendre la présence d'un « $\mathrm{i}$ » dans l'orthographe « oi » de ce genre de groupes dans les dialectes parlers d'oui. (L1)

Suit la conjugaison du verbe être illustrée par une phrase entendue par hasard, la seule apparaissant dans cet échange épistolaire en dehors de saynètes racontées par l'abbé Dourlen (L4, L5) :

Ôz étemmes sur un morchieu d'bous.

Ozetém surỹ mórchy dbw.

Nous étions sur un morceau de bois. (L1)

Les remarques sur le vocalisme concernent la réalisation des nasales et l'antériorisation des labiales : $/ \mathrm{u} /$ et $/ \mathrm{o} />/ \varnothing /$ ou $/ \mathrm{o} /$ [Martinet 1969]. Le système de transcription qu'utilise Pichon n'est pas l'Alphabet Phonétique International, établi en 1886 par P. Passy, qui était réservé à l'enseignement des langues vivantes. De leur côté, les dialectologues se partageaient entre :

- l'alphabet Böhmer-Ascoli.

Une première version de cette transcription panromane a été proposée par Eduard Böhmer en 1871 dans le premier volume des Romanische Studien (295-301). Les susceptibilités patriotiques étaient ménagées, l'exposé étant rédigé en latin : «De sonis grammaticis accuratius distiguendis et notandis ». Du fait que Böhmer était le titulaire de la chaire de romanistique de la nouvelle université allemande de Strasbourg, intronisé dans ce poste dès l'annexion, l'hostilité de Pichon à son encontre était évidente. Cet alphabet a été repris et amendé en Italie par Ascoli à partir des propositions de «Trascrizioni» publiées en introduction du premier volume de l'Archivio Glottologico Italiano (XLII-XLVIII) en 1873. En France, elle est employée par Edouard Bourciez [1910] qui, par sa carrière universitaire à Bordeaux (il est enseignant dans une chaire municipale), pouvait conserver ses distances avec les directeurs de Romania.

- l'alphabet Rousselot.

Conçu par le créateur de la phonétique expérimentale en France, l'abbé Rousselot, et présenté dans la première livraison de la Revue des Patois Gallo-Romans (1887), ce système a été utilisé pour la transcription de l'Atlas Linguistique de la France de Gilliéron et Edmont (1902-1910).

A côté de sons réalisés comme en français (un choix qui se retrouve dans les différents alphabets susmentionnés), Pichon transcrit :

$$
\begin{aligned}
& \mathrm{h}=/ \mathrm{j} / \quad-\text { accolé après } / \mathrm{l} / \mathrm{ou} / \mathrm{n} /, \text { indique la mouillure (palatalisation) } \\
& \mathrm{c}=/ \mathrm{J} / \\
& \mathrm{\omega}=/ \mathrm{w} / \\
& \mathrm{w}=/ \mathrm{u} / \\
& \mathrm{y}=/ \varnothing / \\
& \mathrm{y}=/ \mathrm{\partial} /
\end{aligned}
$$

L'accent aigu et l'accent grave notent le degré d'aperture, le tilde la nasalisation.

Un même son peut être transcrit de deux façons : aucune distinction n'est faite entre la majuscule et la minuscule, la première servant à l'initiale. De même, rien ne permet a priori de différencier les sons notés " ê » et " è » $(=/ \varepsilon /)$ d'un côté, " e » et " é » $(=/ \mathrm{e} /)$ de l'autre. Finalement, le choix de Pichon revient à un décalque de l'orthographe française complété par des conventions qui lui sont propres, sans recours ni aux propositions des romanistes ni à des alphabets « universels » (Lepsius (1855), API...). 


\section{Morphologie \\ Après sa conjugaison (L1), le verbe être fait l'objet de ce commentaire :}

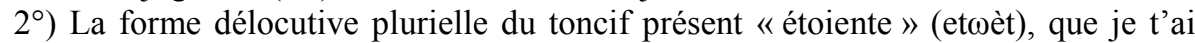
transmise, me paraît inspirée du français, français coulé d'ailleurs dans l'analogie picarde de : «ils peuvnte, ils veulnte » (pývt, výlt).

En effet, en concurrence avec cette forme «étoiente » existe une forme «étenne » (etèn) qui est répond de beaucoup plus près au locutif pluriel de même tiroir :

«étemmes ».

Le « révérentiel » étiez paraît constamment emprunté au français (le phénomène se retrouve identiquement en bourguignon: $\mathrm{Y}$ eume, t'eumes, al eume, y eumons, vous eumez, al eumont), car la politesse exige en quelque sorte, à cette personne, que l'on emploie la langue de la société. (L3)

C'est, dès 1915, la terminologie reprise quinze ans plus tard dans la grammaire: délocutive, toncif, locutif, tiroir, révérentiel. La forme prise par la deuxième personne du pluriel est expliquée par un emploi «révérentiel», quand «la langue de la société »se substitue au patois, en superposant les effets de la diglossie et la sociologie des interactions. Après l'imparfait, la formation du subjonctif et du futur précède une récapitulation du paradigme :

Ceci dit, voici le tableau des désinences, qui te permettra de conjuguer facilement les verbes rosiérois :

Indicatif:

Noncif : selon le cadre

$$
\text { Présent - (ý) ou (z), (ýz) ou (z), (ý) ou (t), (õz), e(z), t(ý). }
$$

Futur - e, o(z), o(t), e(z), õ (t).

Toncif. Présent : $\omega \mathrm{e}(\mathrm{z}), \omega \mathrm{e}(\mathrm{z}), \omega \mathrm{e}(\mathrm{t})$, èm(ýz), h(ẽz), èn(ý)n $\omega$ èt(ý)

Futur : - « idem »-(après l'infinitif, naturell $\left.{ }^{t}\right)$.

Subjonctif Noncif présent : - c(ý), c(ýz), c(ý), chỹ(z), chẽ(z), ct(ý)

Le toncif présent manque.

Subs Verbal Selon le cadre : i, ir, euêr ou r(ý)

Adjf verbal : actif $\tilde{a}(\mathrm{t})$

passif selon le cadre : e, i, u . (L7)

Le «noncif» correspond à une action liée au présent (présent, passé composé...), le « toncif » à une action distante dans le temps (passé ou futur). Les items et les emplois sont collectés au hasard. Le lexique fonctionne à titre d'illustration de la phonétique ou de l'écart par rapport au français. La morphologie, qui concerne presque exclusivement la conjugaison et ne traite en rien de la dérivation, se focalise sur la réalisation des sons sans se donner pour fin de reconstruire le système. Les précautions prises pour circonscrire l'extension géographique des attestations effacent la diversité interne des emplois que Gauchat [1905] avait mise en évidence. Il n'y a aucune indication sur la qualité des informateurs sauf pour l'abbé Dourlen qui s'avère être par ailleurs un puriste dans l'usage du français.

\subsection{Parlers franco-provençaux}

Parce que le but poursuivi par Pichon n'est pas de reconstruire un état dialectal mais seulement d'en mesurer la déviance par rapport au français, toute occasion d'étendre ses inventaires lui paraît bonne à prendre.

Outre que je suis en Picardie, je vis au milieu des troupes du . ${ }^{\mathrm{e}}$ corps, qui est du Dauphiné, de Lyon et de Savoie. Je m'arrange pour faire causer les poilus, et je suis arrivé à cette conclusion : $1^{\circ}$ ) que le savoyard était bien, comme l'affirme Taylor, un patois d'oui. $2^{\circ}$ ) que, même en Dauphiné, la ligne de démarcation oui - oc est beaucoup plus au sud que je ne pensais. Les gens de par là donnent pour limite approximative la ville de Valence. De fait, le «maréchal des logis maréchal», 
Baronnat, avec qui je mange, m'a dit quelques phrases de son patois de la Côté Saint André (Isère), qui ressemblent beaucoup à du bourguignon et sont indéniablement d'oui. Ex : Qu'est-ce que tu fais ici ?
La Côte St André (Isère) :
Tchekkýtýfáitchy?
(itchiere ou itieu)
Gamay (Côte d'or)
Kwaktýfáiki?
(iki). (L6)

- Mon brigadier infirmier est d'Evian et m'a dit quelques mots de son patois

chablaisien qui paraît étrange et amusant.

Suit la transcription de sept noms de fruit afin de récuser la distinction faite entre la langue d'oïl et du franco-provençal. Le rapprochement de celui-ci et du bourguignon paternel permet d'étendre l'aire de rayonnement du français écrit.

On pourrait ajouter cette remarque :

Dire que «chanter» le français différemment, c'est le comprendre différemment, est une hypothèse bien séduisante, mais quelque peu hardie. Je suis en tout cas heureux que tu aies goûté mon observation sur la sensation «chant» dans les accents provinciaux. Quand je parlais de la conversation de Chauffour, je parlais de celle que nous avons eue un jour tous deux en allant chez Jean, et dont l'idée maîtresse était que le langage a, sur un "clavier» d'ailleurs restreint, ses intervalles et sa musique, à valeur purement représentative, à opposer à la musique proprement dite, qui précisément est purement affective. (L14)

Pichon écarte l'hypothèse qu'une différence des accents - c'est-à-dire des dialectes invaliderait la constance des «répartitoires». La langue conserve sa «valeur purement représentative », donc son unité, à travers la diversité de réalisations mélodiques dont la signification est seulement « affective».

\subsection{Occitan et catalan}

\subsubsection{Catalan et biterrois}

L'étude du catalan résulte des hasards d'une rencontre en Champagne au mois de septembre ou octobre 1915 :

Il était une fois un petit Catalan malingre qui pesait 47 kilogs et qui exerçait la profession de vendeur aux Galeries Lafayette, rayon de draperies pour dames. Il se nommait Léophonte. Ajourné deux fois à Paris par les conseils de révision, et voulant partir soldat, il retourna dans son pays où il réussit à obtenir des médecins son départ. Nommé caporal au bout de 4 mois, il conduisait son escouade avec autorité. Le médecin-chef l'a demandé comme cycliste du service de santé, et actuellement il est attaché en cette qualité au médecin du $3^{\mathrm{e}}$ bataillon, c'est-à-dire à moi. Tel est mon professeur de catalan.

Je lui ai fait lire le texte catalan que tu m'as envoyé, le « Pater », et j'ai obtenu ainsi quelques notions de prononciation catalane.

Le $\mathrm{V}$ se prononce $\mathrm{b}$. Il est de tradition d'écrire tantôt $\mathrm{v}$, tantôt $\mathrm{b}$; mais on prononce toujours $b$, le $\mathrm{v}$ n'existe pas en catalan. Cette absence du phonème $\mathrm{v}$ et son remplacement par b existe aussi, je te l'ai dit, dans les dialectes ouest de la lingualité d'oc, que j'appellerai, si tu veux, septimano-gascons.

Donc, ce caractère phonétique est commun aux lingualités espagnole et catalane et à la «pars septimano-vasconica lingualitatis Oc ». Dans ces conditions, il me paraît très plausible de rapporter à la race ibère la paternité de ce caractère... Il serait intéressant de savoir comment se conduit le basque à cet égard, puisque beaucoup de gens considèrent cette langue comme le reliquat de la langue ibérique. (L9)

La suite de la lettre est un commentaire de la traduction en catalan du Pater Noster lue par Léophonte, avec l'indication ajoutée par Pichon des «voyelles toniques». A des remarques sur l'article, la mouillure et le vocalisme (diphtongues), Pichon ajoute la conjugaison du verbe « manger » en catalan et en biterrois (grâce aux informations fournies 
par un autre soldat, Giaselli) et un vocabulaire de dix-huit mots français (les animaux de la ferme) avec leur traduction dans les deux langues. Le courrier se conclut par :

Je t'enverrai les jours suivants d'autres listes. Outre l'intérêt propre qu'ont ces comparaisons, elles nous serviront pour donner de bonnes filières collatérales aux mots dans notre futur Dictionnaire. Ecris-moi si ces questions t'intéressent, et si tu es de mon avis quant à la question du B. (L9)

On apprend ainsi que Damourette et Pichon avaient également le projet de composer un dictionnaire. Quant à la question $\mathrm{du} / \mathrm{b} /-\mathrm{v} /$, elle tient son importance aux yeux de Pichon $\mathrm{du}$ fait qu'elle s'étend à la question des races. La L10 ajoute sept noms d'animaux avec comparaison des réalisations en catalan et en bitterois, la L11 trente-deux noms d'arbre et de fruit en biterrois seulement, la L12 «trois temps tiroirs du verbe "canta", je chante, en languedocien, dialecte biterrois ».

\subsubsection{Provençal}

Le 11 novembre, E. Pichon met un terme à ses enquêtes orales et se consacre au provençal à partir de la lecture de $\mathrm{F}$. Mistral :

J'ai reçu Mirèio, et j'ai commencé à lire cet ouvrage. Il y a des passages d'une remarquable fraîcheur, et je crois que si l'auteur s'était donné la peine d'écrire en vers français, il aurait pu faire un beau poème, très original. Il est donc d'autant plus regrettable qu'il ait criminellement délaissé la langue nationale pour ces dialectes dont, souvent, les consonances ridicules gâtent les jolies idées que contiennent les phrases.

D'ailleurs Mistral pousse la noirceur, et aussi la bêtise, jusqu'à railler le français ; à propos d'une chanson provençale qu'il vient de chanter, l'un des personnages dit :

Aro n'en canton de pu novo

En franchimand, ounte s'atrovo

De mot força pu fin. Mai quau i entend quicon?

(Aujourd'hui ils en chantent de plus nouvelles, en français, où l'on trouve des mots beaucoup plus fins. Mais qui y comprend quelque chose ?)

J'ai ouvert un carnet de notes spécial où, à force d'exemples puisés dans "Mireille», je vais me faire une petite grammaire. Je l'apprendrai ainsi en la faisant, et je te transmettrai ensuite ce que j'y aurai observé de plus remarquable.

Pichon rédige un ensemble de remarques sur le correspondant du /a/ post-tonique latin, la formation de l'article défini et du pluriel avec cette conclusion: «En somme, la lingualité d'oc paraît plutôt moins homogène que celle d'oui ». (L13)

Dans L14, sont évoqués la liaison, le traitement des consonnes et du /o/ en finale :

Une loi très générale du provençal, loi qui résulte de mes observations sur

« Mireille », est la suivante :

Le provençal mistralien ne connaît pas d'o atone non final.

Toutes les fois que, même dans une conjugaison, ou une opération morphologique " régulière », l'o deviendrait atone, il passe à w (écrit : ou)

Ex : ragole, je coule, regoulan, nous coulons.

òli, huile ; oulivo, olive ; ouliveto, olivaie.

Si cette loi n'a pas été formulée, je la revendique hautement et il faudra la caser quelque part, fût-ce dans une note, pour m'en assûrer (sic) la priorité. (L14)

L'essentiel des remarques ne concernent pas tant les faits de langue qu'une appréciation littéraire qui revient sur la priorité qui doit être accordée au français :

Le poème de Mireille où Mistral a su peindre les principaux tableaux de Provence, tant pour les lieux que pour les occupations (olives, magnaneries), et où il a su englober les principales légendes populaires du pays est vraiment une œuvre pleine de charme, et je t'assure que je serais tenté d'y donner des louanges sonores, si je n'étais pas peiné extrêmement de la trahison faite au français. Si elle avait été faite 
en vers français, cette œuvre serait épatante, car on y trouve la fraîcheur de certains tableaux homériques (je pense en ce moment à Nausicaa), et cet englobement de légende et de tableaux de coutumes dans le cadre d'une histoire fort simple représente bien le seul cadre où, du moins avant la guerre, on pouvait concevoir, contemporainement, l'épopée, je veux dire le poème épique. Car pour l'épopée lyrique nous avons eu Hugo ! (Ô soldats de l'an II ; la Colonne ; etc..). Ce que je veux dire, c'est que - la question irritante de la langue mise à part, - Mistral a retrouvé le « genre littéraire » du poème épique de longue haleine, que la Franciade, la Henriade et le Lutrin avaient pu faire croire perdu à jamais. Ces grandes qualités du poème de Mistral sont d'ailleurs une aggravation à son crime d'avoir préféré à la langue française un parler moins souple, moins élégant et parfois un peu ridicule. (L14)

On retrouve dans ces lignes l'archaïsme prêté aux langues régionales, jusques et y compris dans leur inspiration formelle et thématique. Le reproche de n'avoir pas employé le français est un point important pour Pichon qui ralliera l'Action française en s'adressant directement à Charles Maurras. Dans un brouillon de sa demande d'adhésion qu'il rédige le 2 avril 1927, il déclare :

Mais je sais Je sais que la 1 [angue] f[rançaise] n'a pas de meilleur défens illustrateur que vous, Monsieur, et que votre régionalisme crois guère que votre régionalisme linguistique - qui m'a $\mathrm{s}[\mathrm{ou}] \mathrm{v}[\mathrm{en}] \mathrm{t}$ inquiété - ne prétend pas plus à lui ôter sa Royauté Spirituelle que votre légitime rég[ionalisme] politique ne prétend à diminuer le pouvoir du Roi diminuer la fonction supérieure du Roi.

Pichon tente ainsi de concilier la contradiction entre la précellence absolue du français et l'appartenance de Maurras au Félibrige, mouvement provençaliste littéraire créé en 1854 par Mistral.

\section{Une politique des langues: de la langue à la race}

\section{A la fin de L14, on lit :}

Je t'ai rapporté ces différents caractères, pour établir, ce que mes observations subséquentes sur le provençal te montreront encore mieux, que dans son esprit, la lingualité d'oc est plus près de celle d'oui que d'aucune autre. Si l'on réfléchit d'autre part à la grande similitude - (prétendue empruntée, ce dont je doute) - des constructions armoricaines et françaises, on arrive à penser qu'il y a véritablement une unité d'esprit linguistique dans les parlers de France [...]. L'on touche une fois de plus la réalité concrète de la Patrie. Je crois avoir déjà suggéré une fois le profond intérêt qu'il y aurait à étudier l'alsacien quant à sa structure morphologique, syntactique et sémantique. (L14)

Cette prise de position peut être mise en regard de la thèse eurasiste de Troubetzkoy [Sériot 1996]. Contre l'idéologie comparatiste qui postulait des peuples identifiés à leur langue - suivant en cela le modèle de constitution des identités nationales qui a prévalu au $\mathrm{XIX}^{\mathrm{e}}$ siècle [Thiesse 1999] -, Troubetzkoy proposait une convergence progressive, un Sprachbund dont il prenait l'exemple dans les contacts de langues balkaniques. Il en projetait la réalisation sur les frontières de la Russie en dissociant les différentes ethnies de leur rattachement linguistique pour les englober dans un territoire que découpait la superposition d'isoglosses phonologiques et morphologiques. Dans sa vision politique, le rapprochement continu des peuples de l'empire russe leur donnait une nouvelle identité «eurasiste». Cette théorie s'opposait aux déterminants biologiques sous-jacents à la grammaire historique comme à la métaphore américaine du « melting pot».

$\mathrm{Ne}$ reprenant à son compte ni la thèse comparatiste (le postulat d'un peuple originel migrant depuis un foyer primitif et se répandant à travers le sous-continent indien et l'Europe avec une composante variable de métissage), ni les propositions de Troubetzkoy, 
Pichon soutient le principe d'une race première qui se perpétue dans sa «lingualité », intégrant dans le même ensemble des langues de même origine (l'oc et l'oïl) ou de groupes différents (le breton et l'alsacien). On trouve deux pages consacrées à l'alémanique dans ses brouillons.

Ce thème est esquissé dans les considérations sur le ruthène :

Le ruthène n'est d'ailleurs qu'un patois ; la langue littéraire, la langue de la bonne société petite-russienne est, depuis des siècles, le polonais, car pendant des siècles, les Ruthènes ont été loyaux citoyens de la Pologne. Actuellement encore, le ruthène, malgré les efforts des Moscovites, est à peine autre chose qu'un patois. En effet, après que Catherine II eut pris la Petite-Russie, les Moscovites inventèrent de ressusciter la langue ruthène pour l'opposer à la langue polonaise ; ils créèrent de toutes pièces (dit Mickaniewski), et (en tous cas) entretinrent un mouvement national ruthène, comparable au mouvement national provençal qu'on pourrait artificiellement tenter de créer en France. (L2)

La conclusion sur ce point est sans appel: «la Russie n'est pas une quant à la nationalité (la France et l'Espagne, et peut-être l'Italie sont les seuls états vraiment nationaux). » (L2) Ces éléments confortent ce qui est suggéré concernant la race française :

$\S 1$ - Question de la race et du milieu : Jamais ni Edmond Perrier ni moi n'avons voulu nier absolument les migrations ethniques. Influence du milieu sur la race ne signifie pas forcément autochtonie de la race ! Ce que cela signifie, le voici :

Soit une race qui se déplace, et que nous concevons indépendamment même de tout mélange avec une autre. D'une part, elle apportera dans un nouvel habitat des caractères héréditaires permanents qu'elle tiendra de l'influence de son habitat ancien ; et parmi ces caractères, sa langue, son mode de pensée. D'autre part, son nouvel habitat réagira sur elle, la modifiera en certains points, lui apportera de nouveaux caractères. (Mais en particulier, ces conditions surtout physiques agiront plutôt sur le physique de cette race et au minimum sur sa mentalité et sa langue).

(...) C'est cette prédominance ethnique des indigènes qui est, beaucoup plus que l'habitat, (du moins je le crois) le facteur du changement de la langue, par ex. de la transformation du latin en français. Il s'agit alors beaucoup plus, en effet, à mon sens de Gaulois parlant le latin selon leur mentalité gauloise que de Latins modifiant leur esprit et leur langue sous l'influence de l'habitat gaulois. Tu t'aperçois donc que je considère que ce qu'il y a peut-être, de plus constant dans une race, c'est $\underline{\mathrm{sa}}$ mentalité, et son «moule linguistique », sinon sa langue. - Cette idée est un peu révolutionnaire, mais je la crois justifiée et par l'histoire et par la linguistique. (L12)

On découvre dans ces propos une tentative originale - et assez peu scientifique - de faire de la différence des langues l'expression d'une différence des races en présupposant un substrat biologique (E. Perrier présidait aux destinées de la Société Française d'Eugénique). A cet égard, comme pour W. von Humboldt, le basque apparaît comme une possible mise à l'épreuve de la démonstration. La perte de l'opposition phonologique $/ \mathrm{b} / \div$ /v/ est conçue comme un témoin : «Il serait intéressant de savoir comment se conduit le basque à cet égard, puisque beaucoup de gens considèrent cette langue comme le reliquat de la langue ibérique. » (L9), avec une référence à des critères ethniques qui se retrouvent en d'autres endroits de la correspondance :

Rien de neuf à te communiquer sur la question ibérique. Cela sera à revoir, quant à l'anthropologie. Pour nous, ce qui est acquis et que nous pourrons glisser quelque part, c'est le bêtacisme ibérique. (L13)

\section{Conclusion}

Cette correspondance a pour premier intérêt de confirmer la véracité de l'indication chronologique «1911-1927 » portée en sous-titre de Des mots à la pensée dont le premier volume est édité en 1930. On apprend que son dédicataire, Jean Weber, a mis un terme à sa 
collaboration pour prendre la direction du Journal en 1915 et que le projet initial comprenait la rédaction d'un dictionnaire dont ne subsistent que des «Notes de lexicographie », une cinquantaine de pages inégalement remplies dans un carnet de petite dimension.

Ces lettres apportent un éclairage singulier sur la perspective linguistique. Pichon explicite sa conception de la langue comme cadre de pensée et expression d'une même race qui serait présente sur le territoire de la Gaule depuis l'Antiquité. La diversité des parlers ne contrevient pas à une identité biologique qui transparaît dans la convergence des langues d'oc et d'oïl, du breton et de l'alsacien. Si Pichon se démarque des comparatistes allemands, ce n'est pas pour prendre ses distances avec la raciologie puisqu'il accorde à la constitution ethnique la capacité de transcender la diversité des langues. Les critères biologiques conditionnent l'organisation sociale et l'expression langagière.

Ce préjugé se situe à l'inverse des thèses défendues par G. Paris dans la présentation de Romania en 1872 :

La Romania, ou l'union des nations romanes, n'a pas pour base une communauté de race. Quand on parle des races latines on emploie une expression qui manque absolument de justesse : il n'y a pas de races latines. (Paris $1872: 20$ )

Il s'oppose également aux conceptions sociologiques qu'expose A. Meillet dans sa leçon inaugurale au Collège de France [1906]. Au-delà de leur statut diglossique, les patois sont devenus un argument dans une vision raciste dont Pichon cherchera l'illustration jusque dans l'inconscient des locuteurs.

\section{Références bibliographiques}

Ascoli, G. I. (1873). Trascrizioni, Archivio Glottologico Italiano, I, XLII-XLVIII.

Böhmer, E. (1871). De sonis grammaticis accuratius distiguendis et notandis, Romanische Studien, I, 295-301.

Bourciez, E. (1910). Éléments de linguistique romane, Paris : C. Klincksieck.

Brunot, F. (1905-1937). Histoire de la langue française, Paris : A. Colin.

Damourette, J. \& Pichon, E. (1930-1950). Des mots à la pensée. Essai de grammaire de la langue française, Paris : Éditions d'Artrey.

Gauchat, L. (1905). L'unité phonétique dans le patois d'une commune, in Aus romanischen Sprachen und Literaturen. Festschrift Heinrich Morf, Halle : Niemeyer, 175-232.

Gilléron, J. \& Edmont, E. (1902-1910). Atlas linguistique de la France, Paris, Champion.

Lepsius, R. (1855) Das allgemeine linguistische Alphabet, Berlin : Verlag von Wilhelm Hertz.

Mannoni, J. (2016). Catalogue Edouard Pichon, http://des.livres.autour.pagespersoorange.fr/cat20damourette-pichon.pdf [consulté le 15 décembre 2017]

Martinet, A. (1969) «C'est jeuli le Mareuc », in Le Français sans fard, Paris, PUF : 191-219.

Meillet, A. (1906) L'état actuel des études de linguistique générale, "Leçon d'ouverture du cours de Grammaire comparée au Collège de France », Chartres : Imprimerie Durand.

Milner, J.-C. (1978) L'Amour de la langue, Paris : Seuil.

Muni Toke, V. (2013). La grammaire nationale selon Damourette et Pichon. 1911-1939. L'invention du locuteur, Lyon, : ENS Editions.

Nyrop, K. (1899-1930). Grammaire historique de la langue française, Copenhague : Gyldendal.

Paris, G. (1872). « Romani, Romania, lingua romana, romancium », Romania, I, 1-22.

Paris, G. (1888). «Les parlers de France », Revue des Patois Gallo-Romans, 2, 161-175.

Portine, H. (ed.) (1996). Actualité de Jacques Damourette et Edouard Pichon, Langages, 124.

Rousselot, J.-P. (1887). Introduction à l'étude des patois, Revue des Patois Gallo-Romans, I, 1-22.

Sériot, P. (1996). N. S. Troubetzkoy, l'Europe et l'humanité, Liège : Mardaga.

Thiesse, A.-M. (1999). La Création des identités nationales, Paris : Seuil. 